\title{
Multiplicity, Instability, and SCF Convergence Problems in Hartree-Fock Solutions
}

\author{
L. E. DARDENNE, ${ }^{1, *}$ N. MAKIUCHI, ${ }^{1}$ L. A. C. MALBOUISSON, ${ }^{2}$ \\ J. D. M. VIANNA ${ }^{1,2}$ \\ ${ }^{1}$ Instituto de Física, Universidade de Brasília, 70910-900 Brasília, DF, Brazil \\ ${ }^{2}$ Instituto de Física, Universidade Federal de Bahia, Campus de Ondina, 40210-340 Salvador, BA, Brazil
}

Received 10 November 1998; revised 19 July 1999; accepted 31 August 1999

\begin{abstract}
We present a study of the instability and convergence of Hartree-Fock (HF) ab initio solutions for the diatomic systems $\mathrm{H}_{2}, \mathrm{LiH}, \mathrm{CH}, \mathrm{C}_{2}$, and $\mathrm{N}_{2}$. In our study, we consider real molecular orbitals (MOs) and analyze the classes of single-determinant functions associated to Hartree-Fock-Roothaan (HFR) and Hartree-Fock-Pople-Nesbet (HFPN) equations. To determine the multiple HF solutions, we used either an SCF iterative procedure with aufbau and non-aufbau ordering rules or the algebraic method (AM). Stability conditions were determined using TICS and ASDW stability matrices, derived from the maximum and minimum method of functions (MMF). We examined the relationship between pure SCF convergence criterion with the aufbau ordering rule, and the classification of the HF solution as an extremum point in its respective class of functions. Our results show that (i) in a pure converged SCF calculation, with the aufbau ordering rule, the solutions are not necessarily classified as a minimum of the HF functional with respect to the TICS or ASDW classes of solutions, and (ii) for all studied systems, we obtained local minimum points associated only with the aufbau rule and the solutions of lower energies. C C 2000 John Wiley \& Sons, Inc. Int J Quant Chem 76: 600-610, 2000
\end{abstract}

Key words: multiplicity; instability; SCF convergence; Hartree-Fock solutions; algebraic method 


\section{Introduction}

I nstabilities in Hartree-Fock (HF) solutions and their consequences have been studied by several authors in many versions [1-3]. Extremum sufficient conditions for the HF problem were formulated initially by Thouless [1] and Adams [2]. Cizek and Paldus [4] introduced singlet and triplet instability concepts. Löwdin et al. [5] deduced stability criteria for the projected HF problem and related their approach with Cizek and Paldus's results. Fukutome [6], using group theory, proposed a general classification for HF instabilities. Also, it is possible to obtain all classes of the HF instabilities using concepts of functional analysis $[7,8]$. On the other hand, the usual procedure to resolve the HF equation, the self-consistent field (SCF) method, in some cases, presents convergence problems. Stanton [9] introduced an intrinsic numerical convergence criterion for the pure closed-shell SCF calculations, that is, SCF calculation without use of any algorithm to enforce convergence.

In this context, Facelli and Contreras [10] presented a relation between instabilities of the closedshell HF wave functions and Stanton's criterion [9]. In a subsequent article [11], those authors showed a relation among the convergence in the unrestricted HF method (UHF), SCF calculations, and Löwdin's stability condition [5]. In this work, we present some comments and numerical results about this question. This article is organized as follows: In Section 2, we present the methodology that we have used and a résumé of the theory; Section 3 contains our results, and Section 4 , our conclusions.

\section{Theory}

\subsection{MULTIPLICITY OF HF SOLUTIONS}

The HF equation is a nonlinear equation owing to its SCF character and, therefore, it presents, in principle, several solutions. Besides the usual SCF method for solving the HF equation, in LCAO (linear combination of atomic orbitals) formulation, there is an equivalent procedure, the algebraic method (AM) [12], developed for solving an algebraic system of nonlinear equations in the LCAO coefficients $C_{u u^{\prime}}$. The general AM procedure is a computationally different approach, which, unlike the usual iterative procedure SCF, does not use ordering rules. For the Hartree-Fock-Roothaan (HFR) closed-shell problem, it is based on the following considerations: (i) The equations of the algebraic system are

$$
\begin{aligned}
F_{\mu^{\prime}, v^{\prime}}=\sum_{\mu \nu}^{m} C_{\mu \mu^{\prime}} & C_{\nu \nu^{\prime}} h_{\mu \nu}+\sum_{i=1}^{N} \sum_{\mu \nu \lambda \sigma}^{m} C_{\mu \mu^{\prime}} C_{\lambda i} C_{\nu \nu^{\prime}} C_{\sigma i} \\
& \times[2\langle\mu \lambda \mid \nu \sigma\rangle-\langle\mu \lambda \mid \sigma \nu\rangle]=0,
\end{aligned}
$$

with $\mu^{\prime}<v^{\prime}$. The symbol $i$ stands for occupied molecular orbitals (MOs) and

$$
\begin{aligned}
S_{\mu^{\prime}, v^{\prime}}=\sum_{\mu \nu}^{m} C_{\mu \mu^{\prime}} C_{\nu v^{\prime}} S_{\mu \nu}=\delta_{\mu^{\prime} v^{\prime}} \\
\mu^{\prime} \leq v^{\prime} ; \quad \mu^{\prime}, v^{\prime}=1, \ldots, m,
\end{aligned}
$$

where $S_{\mu \nu}, h_{\mu \nu}$, and $\langle\mu \lambda \mid v \sigma\rangle$ are the overlap integral, one-electron integral, and two-electron integral, respectively; $\mu$ denotes atomic orbitals (AOs), and $\mu^{\prime}$, (MOs). (ii) The orbital energies $\varepsilon_{\mu^{\prime}}$ are given by the diagonal elements $F_{\mu^{\prime}, \mu^{\prime}}$. (iii) Equations (2.1) and (2.2) constitute a system of $\mathrm{m}^{2}$ independent nonlinear algebraic equations in the $m^{2}$ variables $C_{u u^{\prime}}$; this algebraic equation system is equivalent to the canonical integrodifferential HFR equations and can be solved using, for instance, a quadratically convergent Newton-like method [15]. Each solution of this system corresponds to a set of $m$ vectors $\mathbf{C}_{\mu^{\prime}}=\left(C_{1 \mu^{\prime}}, C_{2 \mu^{\prime}}, \ldots, C_{m \mu^{\prime}}\right)$, where we have $\mu^{\prime}=1,2, \ldots, N$ for the occupied molecular orbitals and $\mu^{\prime}=N+1, \ldots, m$ for the respective set of virtual molecular orbitals. The $N$ occupied molecular orbitals are not necessarily related to the lowest orbital energies $\varepsilon_{\mu^{\prime}}$ or, in other words, do not necessarily satisfy the aufbau principle. (iv) We define the occupancy scheme in the AM procedure by the relative order of the $N$ occupied $\mathrm{MO}$ energies in the increasing ordered set of the $m \mathrm{MO}$ (occupied + virtual) energies. Unlike the SCF procedure, the occupancy scheme in the AM procedure is only known at the end of the calculus.

For the Hartree-Fock-Pople-Nesbet (HFPN) equations, the AM procedure gives a similar algebraic system [13] with $2 m^{2}$ independent nonlinear equations. The occupancy scheme for each set of spin molecular orbitals is defined in the same way as that for the HFR case.

In the usual iterative SCF procedure, it is also possible to obtain canonical converged RHF or UHF solutions which do not obey the aufbau principle of occupation. It is possible to obtain these non-aufbau solutions if at each step of the iterative procedure we systematically leave unfilled one or more MOs with a lower orbital energy and replace them by others with greater orbital energies. The final scheme 
of occupation is determined by the ordering rule (based on the $m$ relative MO orbital energies) used to fill the occupied moleculars which are used to construct the Fock matrix at each step of the iterative procedure. This simple strategy to obtain self-consistent non-aufbau solutions has the drawback to generate, in many cases, a divergent or oscillatory process. We can take advantage of these nonconvergent processes using their nonconverged LCAO coefficients as starting points for the AM procedure. It is important to note that different converged solutions can be obtained if we start the SCF or the AM procedure with different initial guesses for the LCAO coefficients. In this work, the different initial guesses for the AM procedure are taken as the nonconverged LCAO coefficients generated at some step of nonconvergent SCF procedures with different ordering rules. In this way, it is possible to obtain a set of converged solutions with different occupation schemes.

As related in the literature, there are HF solutions which are not obtained using the SCF procedure (pure or not) but they can be determined using the AM procedure. So, to analyze some results presented by Stanton, Contreras, and Facelli about the SCF solutions, we shall consider, in the calculations, both the SCF and AM procedures $[12,13]$.

\subsection{STABILITY AND CONVERGENCE OF HF SOLUTIONS}

The HF equation is a necessary condition equation (first-order necessary condition) for the existence of extremum in a variational problem. Specifically, any HF solution should be a minimum, maximum, or saddle point of the electronic energy functional:

$$
E(\Psi)=\frac{\langle\Psi|\widehat{H}| \Psi\rangle}{\langle\Psi \mid \Psi\rangle}
$$

where $\widehat{H}$ is the Hamiltonian of the system and $\Psi$ is a trial monodeterminantal function.

The kind of HF extremum is determined from sufficient conditions (second-order conditions), which can be presented as a matrix called stability matrix. In this context, the kind of extremum is determined by an analysis of the signs of stability matrix eigenvalues $\left\{\lambda_{i}\right\}$ associated with the HF solution. If all $\lambda_{i}>0$, then the extremum is a local minimum; if all $\lambda_{i}<0$, then the extremum is a local maximum. Otherwise, the extremum is a saddle point.

Stanton's criterion [9] for intrinsic numerical convergence in closed-shell SCF calculation considers a matrix $\mathbf{M}$ and a matrix $\mathbf{Q}$, given in Stanton's notation, as

$$
\begin{aligned}
& \mathbf{M}_{2, i j}=\sum_{r, s} \mathbf{Q}_{i j ; r s} \mathbf{M}_{1, r s} \\
& \mathbf{Q}_{i j ; r s}=-\frac{(4\langle i r \mid j s\rangle-\langle i j \mid r s\rangle-\langle i j \mid s r\rangle)}{\sqrt{\left(\epsilon_{i}-\epsilon_{j}\right)\left(\epsilon_{r}-\epsilon_{s}\right)}},
\end{aligned}
$$

where the $\mathbf{Q}$ matrix is formed by the exact occupied MOs $i$ and $r$ and by the virtual MOs $j$ and $s$, respectively. The matrix $\mathbf{M}_{n}$ represents the error in the wave function at the $n$-th cycle of the SCF procedure. More specifically, $\mathbf{M}_{n, r s}$ represents the error due to the contamination of the occupied orbital $r$ by the virtual orbital $s$ at the $n$-th SCF iteration. Equation (2.4) governs the propagation of error from one SCF iteration to the next. The convergence criterion is given in terms of the largest eigenvalue $\left|\lambda_{\max }\right|$ of the matrix $Q$. The SCF procedure is intrinsically convergent if $\left|\lambda_{\max }\right|<1$.

In the comment of Facelli and Contreras [10] about Stanton's article, those authors have established a relation between the eigenvalues of $\mathbf{Q}$, given by Eq. (2.5), and the eigenvalues of the stability matrix E (Cizek-Paldus singlet stability matrix [4]), which is written, in Stanton's notation, as

$$
\begin{aligned}
\mathbf{E}_{i j ; r s}=\left(\epsilon_{i}-\epsilon_{j}\right) \delta_{i r} \delta_{j s} & \\
& +(4\langle i r \mid j s\rangle-\langle i j \mid r s\rangle-\langle i j \mid s r\rangle) .
\end{aligned}
$$

Facelli and Contreras [10] affirmed that when the Stanton's convergence criterion [Eq. (2.5)] is satisfied, the singlet stability condition [Eq. (2.6)] is also satisfied. To obtain this result, they supposed that the aufbau principle is followed and wrote the matrix $\mathbf{Q}$ as the product between a symmetric matrix $\mathbf{S}$ and a diagonal positive matrix $\mathbf{D}$ given, respectively, by

$$
\begin{aligned}
& \mathbf{S}_{m k ; r s}=-(4\langle m r \mid k s\rangle-\langle m k \mid r s\rangle-\langle m k \mid s r\rangle) \\
& \mathbf{D}_{i j ; m k}=\left(\epsilon_{i}-\epsilon_{j}\right)^{-1} \delta_{j k} \delta_{i m} .
\end{aligned}
$$

In terms of $\mathbf{D}$ and $\mathbf{S}$, the following expressions are obtained for $\mathbf{Q}$ and $\mathbf{E}$ :

$$
\begin{aligned}
& \mathbf{Q}=\mathbf{D}^{1 / 2} \mathbf{S D}^{1 / 2} \\
& \mathbf{E}=\mathbf{D}^{-1 / 2}\left(1-\mathbf{D}^{1 / 2} \mathbf{S D}^{1 / 2}\right) \mathbf{D}^{-1 / 2} .
\end{aligned}
$$

Hence, they noted that if all eigenvalues of $\mathbf{D}^{1 / 2} \mathbf{S D}^{1 / 2}$ are smaller than unity in absolute value, those corresponding to the matrix $\mathbf{1}-\mathbf{D}^{1 / 2} \mathbf{S D}^{1 / 2}$ will be positive, and the matrix $\mathbf{E}$ will have positive eigenvalues also, since a pre- and post-multiplication by $\mathbf{D}^{-1 / 2}$ does not modify the sign of its eigenvalues. In consequence, as their result becomes a condition for the existence of a stable singlet 
closed-shell SCF wave function, they concluded that "if the classical algorithm converges, then one can be sure that the corresponding closed-shell energy is a minimum in allowed space of singlet functions." In a second article [11], those authors extended their conclusion for a general one-determinantal wave function which satisfies the aufbau principle.

In his article, Stanton [9] made a series of symmetry considerations, not mentioned in the work of Contreras and Facelli, about the choice of SCF starting orbitals, the symmetry of the final solution, and his criterion for intrinsic numerical convergence. If the desired final solution has completely broken symmetry or if we start the SCF procedure with initial orbitals without any symmetry character, his criterion holds with full rigor. The final SCF will diverge unless $\left|\lambda_{\max }\right|$ of its respective $\mathbf{Q}$ matrix is less than 1 . If the final SCF solution presents any symmetry in its MOs, it is always possible to make a symmetry blocking decomposition of the $\mathbf{Q}$ matrix. Then, in the $M_{n, r s}$ error matrix, there will be blocks connecting the errors propagated between orbitals of the same symmetry species and blocks connecting the propagation of errors between orbitals of different symmetries or, in other words, symmetry mixing blocks. In this case, if we start the SCF procedure with orbitals symmetrically adapted, it is equivalent to start with zero elements in the mixing blocks. These errors will continue to be zero during the interative procedure due to the resultant symmetry of the Fock matrix. The consequence of this last consideration is that the presence of $\mathbf{Q}$ eigenvalues greater than 1 in absolute value are not implicated in an intrinsically divergent process if we start the SCF procedure with symmetrically adapted orbitals, or containing some species of symmetry, and these eigenvalues are associated with the correspondent symmetry mixing blocks. It is perfectly possible that in a pure converged SCF procedure an eigenvalue associated to a symmetry mixing block of the $\mathbf{Q}$ matrix has a value greater than 1 . It is easy to see in Eq. (2.10) that, in this case, the stability matrix $E$ will have at least one eigenvalue less than 1 and, consequently, the final solution will be not classified as a minimum in the respective space of functions associated to the stability matrix.

In the following section, to analyze the Facelli and Contreras conclusion, we present a numerical study about the relation between the SCF convergence problem and the stability of the HF real solution in HFR and HFPN calculations without use of spacial symmetry restrictions.

\section{Results}

We present results for ab initio calculations. We use the GAMESS [14] code in conjunction with our own computer codes [12,13,15] for the AM calculations and stability analysis. We consider $\mathrm{H}_{2}, \mathrm{LiH}$, $\mathrm{CH}, \mathrm{C}_{2}$, and $\mathrm{N}_{2}$ diatomic systems, at their equilibrium interatomic distances, without use of any point symmetry condition. For closed-shell systems, the equilibrium interatomic distance was obtained using the RHF method, and for open-shell systems, using the UHF method. For $\mathrm{H}_{2}$ systems, we used a triple-zeta basis with six basis functions, and for other systems, we used a minimal basis STO-6G.

Our studies concern the instabilities associated with two classes of solutions in Fukutome classification [6]: the time-reversal invariant closed-shell solutions (TICS) associated with the HFR functional, which are eigenfunctions of $S_{z}$ and $S^{2}$ operators, and the axial spin density wave solutions (ASDW) associated with the HFPN functional, which are eigenfunctions of $S_{z}$, but not of $S^{2}$ operators. These two classes are associated with one-determinantal solutions formed by real spin MOs and are connected only to different symmetry restrictions upon spin. No spacial symmetry restrictions in the Fock matrices are imposed. An RHF solution can be regarded as a special case of a UHF solution when the $\alpha$ set of the LCAO coefficients have the same value of the $\beta$ set and we can consider the space of functions associated with the HFR functional as a subspace of the space associated with the HFPN functional. A solution belonging to the TICS/RHF class can be characterized as a stationary point within the HFR functional space (by the TICS stability matrix) or within the HFPN functional space (by the ASDW stability matrix).

Our calculations were done using the pure SCF procedure with aufbau and non-aufbau (implemented by us in the GAMESS code) schemes (see Section 2.1), for HFR and HFPN equations. For initial SCF MOs, we used those obtained from the GAMESS's Extended Huckel option, which normally leads to a set of initial molecular orbitals with $\sigma$ or $\pi$ symmetry character. The accuracy of all SCF calculations is less than $10^{-5}$ in the density matrix. For the solutions which present a saddle-point character, the accuracy was increased to $10^{-10}$. In some cases, where pure SCF procedure with the aufbau scheme did not reach convergent solutions, we used routines to enforce SCF convergence. In other cases, when an SCF calculation did not reach convergent 
TABLE I

Multiple UHF/HFPN solutions for $\mathrm{H}_{2}$.

\begin{tabular}{cccccc}
\hline Solution & Total energy & Occupancy & Extremum & $\left\langle S^{2}\right\rangle$ & Method \\
\hline 1 & -1.128029 & $(1)(1)$ & Minimum & 0.0 & SCF \\
2 & -0.690646 & $(2)(1)$ & SP (1) & 1.0 & AM \\
3 & -0.555071 & $(2)(1)$ & SP (2) & 0.977 & AM \\
4 & -0.279593 & SP (3) $(1)$ & SP (2) & 0.0 & AM \\
5 & -0.060009 & $(2)(2)$ & SCF \\
\hline
\end{tabular}

Interatomic distance $R e=0.7315368 \AA$. Total energy is given in Hartrees. Occupancy means the MO occupancy scheme-in the first parentheses, alpha molecular spin-orbital occupancy, and in the second parentheses, beta molecular spin-orbital occupancy. The ASDW stability matrix is used to characterize the extremum. The notation $\operatorname{SP}(X)$ means a saddle point with $X$ stability matrix eigenvalues less than zero.

solutions or presented an oscillatory behavior, we used the AM discussed above with the nonconvergent SCF-LCAO coefficients as a starting point. The accuracy of all AM calculations is less than $10^{-7}$ in the LCAO expansion coefficients. We used the stability matrices for TICS and ASDW solutions [6-8] in order to characterize the nature of the HF solution as an extremum point. For $\mathrm{H}_{2}, \mathrm{LiH}, \mathrm{C}_{2}$, and $\mathrm{N}_{2}$ closed-shell systems, we calculated $\mathbf{Q}$ Stanton's matrix eigenvalues of the solutions belonging to TICS class, found by an aufbau RHF/SCF procedure, pure or not. We took care of determining the solutions with an accuracy of $10^{-10}$ in density matrix in order to apply Stanton's criterion.

The results below present several converged HF solutions that we have found. In all tables, the total energy is given in Hartrees, and occupancy scheme means $\mathrm{MO}$ occupancy, where in the first parentheses we present alpha molecular spin orbital occupancy, and, in the second parentheses, the correspondent beta molecular spin orbital occupation (see Section 2.1). The notation $\mathrm{SP}(X)$ means a saddle point with $X$ stability matrix eigenvalues less than zero. Solutions classified as minimum* have all eigenvalues of the stability matrix greater than zero except one which is zero (a zero eigenvalue indicates that there exists a parameter for which the energy value is independent at least up to the second order [3]).

We present in Table I multiple UHF/HFPN solutions for $\mathrm{H}_{2}$ with $\left\langle S_{z}\right\rangle=0.0$ and interatomic distance $R e=0.7315368 \AA$. The characterization of the type of extremum for each solution was made using the corresponding ASDW stability matrix. Solutions 1 and 5 belong to TICS class (see Table II). The minimum aufbau solutions were obtained using pure SCF procedure.

In Table III we present some multiple UHF/ HFPN solutions for $\mathrm{LiH}$, with $\left\langle S_{z}\right\rangle=0.0$ and interatomic distance $R e=1.5065913 \AA$. The characterization of the type of extremum for each solution was made using the corresponding ASDW stability matrix. Again, we found that the minimum extremum point is an aufbau solution and was determined using pure SCF calculation for the HFPN equations. But for this system, we found two other aufbau solutions (see solutions 2 and 3) using the AM procedure; these solutions are identified as saddle points. Solutions 6, 7, and 9 belong to the TICS class. They were obtained by the pure RHF-SCF method and

TABLE II

Multiple RHF solutions for $\mathrm{H}_{2}$.

\begin{tabular}{ccclcc}
\hline Solution & Total energy & Occupancy & Extremum & $\left\langle S^{2}\right\rangle$ & Method \\
\hline 1 & -1.128029 & $(1)$ & Minimum & 0.0 & SCF \\
2 & -0.060009 & $(2)$ & SP (1) & 0.0 & SCF \\
\hline
\end{tabular}

Interatomic distance $R e=0.7315368 \AA$. Total energy is given in Hartrees. Occupancy means MO occupancy scheme. The TICS stability matrix is used to characterize the extremum. The notation $\operatorname{SP}(X)$ means a saddle point with $X$ stability matrix eigenvalues less than zero. 
TABLE III Multiple UHF/HFPN solutions for $\mathrm{LiH},\left\langle S_{z}\right\rangle=\mathbf{0 . 0}$.

\begin{tabular}{|c|c|c|c|c|c|}
\hline Solution & Total energy & Occupancy & Extremum & $\left\langle S^{2}\right\rangle$ & Method \\
\hline 1 & -7.953470 & $(1,2)(1,2)$ & Minimum & 0.0 & SCF \\
\hline 2 & -7.835055 & $(1,2)(1,2)$ & $\mathrm{SP}(1)$ & 0.924 & $\mathrm{AM}$ \\
\hline 3 & -7.792617 & $(1,2)(1,2)$ & SP (2) & 1.0 & AM \\
\hline 4 & -7.320230 & $(1,3)(1,3)$ & SP (3) & 1.0 & AM \\
\hline 5 & -7.317940 & $(1,3)(1,3)$ & SP (4) & 1.0 & $\mathrm{AM}$ \\
\hline 6 & -7.317221 & $(1,3)(1,3)$ & SP (4) & 0.0 & SCF \\
\hline 7 & -7.284190 & $(1,3)(1,3)$ & SP (4) & 0.0 & $\mathrm{AM}$ \\
\hline 8 & -7.133688 & $(1,3)(1,3)$ & SP (6) & 1.0 & AM \\
\hline 9 & -6.852158 & $(1,6)(1,6)$ & SP (4) & 0.0 & $\mathrm{AM}$ \\
\hline 10 & -5.873923 & $(2,3)(1,2)$ & SP (2) & 1.0 & SCF \\
\hline 11 & -5.802609 & $(2,3)(1,2)$ & SP (3) & 1.032 & $\mathrm{AM}$ \\
\hline
\end{tabular}

Interatomic distance $R e=1.5065913 \AA$. Total energy is given in Hartrees. Occupancy means MO occupancy scheme-in the first parentheses, alpha molecular spin-orbital occupancy, and in the second parentheses, beta molecular spin-orbital occupancy. The ASDW stability matrix is used to characterize the extremum. The notation $\operatorname{SP}(X)$ means a saddle point with $X$ stability matrix eigenvalues less than zero.

the AM procedure and characterized using the TICS stability matrix (Table IV).

For $\mathrm{H}_{2}$ and $\mathrm{LiH}$ aufbau SCF solutions belonging to the TICS class, we found that all $\mathbf{Q}$ Stanton's matrix eigenvalues are less than 1 in absolute value. In these cases, there is not any intrinsic numerical divergence. This is in accordance with the fact that these solutions were found by a pure SCF procedure.

In Table V, we present some UHF/HFPN solutions for $\mathrm{LiH}$, with $\left\langle S_{z}\right\rangle=1.0$ and the same interatomic distance as above; these solutions belong to the ASDW class. Result 1 is a minimum aufbau solution and was determined by the pure SCF method; solution 2 also is an aufbau solution but it has been obtained by the AM procedure and is classified as a saddle point. For both, $\left\langle S_{z}\right\rangle=0.0$ and $\left\langle S_{z}\right\rangle=1.0$, we obtained only one minimum extremum point for each class of solutions.

Table VI contains some multiple UHF solutions for $\mathrm{CH}$, with $\left\langle S_{z}\right\rangle=0.5$ and interatomic distance $\operatorname{Re}=1.1380338 \AA$; these solutions belong to the ASDW class and were obtained by HFPN equations. We note that in this case the pure SCF method gives an aufbau solution which is not characterized as a minimum, in the ASDW class of solutions, but as a saddle point. This result contradicts Facelli and Contreras' conclusion [11]. Using the AM procedure, we obtained another aufbau solution with energy less than that determined from pure SCF method and this AM solution is classified as a minimum. It is possible to find this minimum using the SCF method, but the only way we found to do this is by the utilization of algorithms to enforce

TABLE IV

Multiple RHF solutions for $\mathrm{LiH},\left\langle S_{\mathbf{z}}\right\rangle=\mathbf{0 . 0}$.

\begin{tabular}{cccccc}
\hline Solution & Total energy & Occupancy & Extremum & $\left\langle S^{2}\right\rangle$ & Method \\
\hline 1 & -7.953470 & $(12)$ & Minimum & 0.0 & SCF \\
2 & -7.317221 & $(13)$ & SP (1) & 0.0 & SCF \\
3 & -7.284190 & $(13)$ & SP (1) & 0.0 & AM \\
4 & -6.852158 & $(16)$ & SP (4) & 0.0 & AM \\
\hline
\end{tabular}

Interatomic distance $R e=1.5065913 \AA$. Total energy is given in Hartrees. Occupancy means MO occupancy scheme. The TICS stability matrix is used to characterize the extremum. The notation $\operatorname{SP}(X)$ means a saddle point with $X$ stability matrix eigenvalues less than zero. 
TABLE V

Multiple UHF/HFPN solutions for $\mathrm{LiH},\left\langle S_{z}\right\rangle=1.0$.

\begin{tabular}{cccccc}
\hline Solution & Total energy & Occupancy & Extremum & $\left\langle S^{2}\right\rangle$ & Method \\
\hline 1 & -7.849592 & $(123)(1)$ & Minimum & 2.0 & SCF \\
2 & -7.803801 & $(123)(1)$ & SP (2) & 2.0 & AM \\
3 & -7.375723 & $(134)(1)$ & SP (2) & 2.0 & AM \\
4 & -7.151784 & $(135)(1)$ & SP (5) & 2.0 & AM \\
\hline
\end{tabular}

Interatomic distance $R e=1.5065913 \AA$. Total energy is given in Hartrees. Occupancy means MO occupancy scheme-in the first parentheses, alpha molecular spin-orbital occupancy, and in the second parentheses, beta molecular spin-orbital occupancy. The ASDW stability matrix is used to characterize the extremum. The notation $\operatorname{SP}(X)$ means a saddle point with $X$ stability matrix eigenvalues less than zero.

the convergence in conjunction with a very specific choice of the starting LCAO coefficients. Other solutions were obtained by the AM procedure and all are classified as saddle points. Solution 8 presents a non-aufbau occupancy and a lower energy than that of solution 9 with aufbau occupancy.

Table VII refers to the $\mathrm{CH}$ system and UHF/ HFPN equations, but with $\left\langle S_{z}\right\rangle=1.5$ and the same interatomic distance $R e=1.1380338 \AA$. Here, the pure SCF method leads to an aufbau solution which is classified as a minimum and presents an energy lower than that of any solution obtained with $\left\langle S_{z}\right\rangle=0.5$. The AM procedure exhibits other aufbau solutions (see solutions 2, 3, and 7) classified as saddle points. It is interesting to note that the nonaufbau solutions 4 and 6 present an energy lower than that of the aufbau solutions 5 and 7, respectively.
The fact that we have found, for systems $\mathrm{LiH}$ and $\mathrm{CH}$, many converged solutions which present an aufbau occupancy using the AM procedure does not signify that these solutions cannot be obtained by the SCF procedure. It is probable that with a specific choice for the initial guess (like different filling schemes for the $\pi$-orbitals) several among these solutions can be recovered using a pure aufbau SCF procedure. Our results showing that these solutions were obtained by the AM procedure reflect the methodology which we used (described at the end of Section 2.1), where unconverged solutions using the SCF procedure with different ordering rules are used as starting points for the AM procedure.

In Table VIII, we present multiple UHF/HFPN solutions for $C_{2}$, with $\left\langle S_{z}\right\rangle=0.0$, interatomic distance $R e=1.2337470 \AA$, and accuracy less than $10^{-8}$ in the density matrix. In all calculations, we

TABLE VI

Multiple UHF/HFPN solutions for $\mathrm{CH},\left\langle\mathrm{S}_{\mathbf{z}}\right\rangle=0.5$.

\begin{tabular}{|c|c|c|c|c|c|}
\hline Solution & Total energy & Occupancy & Extremum & $\left\langle S^{2}\right\rangle$ & Method \\
\hline 1 & -38.149624 & (1234) (123) & Minimum* & 1.081 & AM \\
\hline 2 & -38.145699 & (1234) (123) & $S P(1)$ & 0.753 & SCF \\
\hline 3 & -38.062958 & (1234) (123) & SP (1) & 1.762 & $\mathrm{AM}$ \\
\hline 4 & -38.058218 & (1234) (123) & SP (2) & 1.809 & AM \\
\hline 5 & -37.990395 & (1234) (123) & SP (2) & 0.762 & AM \\
\hline 6 & -37.799653 & (1234) (123) & SP (3) & 0.908 & AM \\
\hline 7 & -37.672278 & (1234) (123) & SP (4) & 0.750 & AM \\
\hline 8 & -37.654388 & (1234) (124) & SP (2) & 1.766 & AM \\
\hline 9 & -37.639109 & (1234) (123) & SP (3) & 1.750 & AM \\
\hline
\end{tabular}

Equilibrium interatomic distance $R e=1.1380338 \AA$. Total energy is given in Hartrees. Occupancy means MO occupancy schemein the first parentheses, alpha molecular spin-orbital occupancy, and in the second parentheses, beta molecular spin-orbital occupancy. The ASDW stability matrix is used to characterize the extremum. The notation SP $(X)$ means a saddle point with $X$ stability matrix eigenvalues less than zero. 
TABLE VII Multiple UHF/HFPN solutions for $\mathrm{CH},\left\langle S_{z}\right\rangle=1.5$.

\begin{tabular}{|c|c|c|c|c|c|}
\hline Solution & Total energy & Extremum & $\left\langle S^{2}\right\rangle$ & Method & \\
\hline 1 & -38.167209 & $(12345)(12)$ & Minimum & 3.755 & SCF \\
\hline 2 & -37.776728 & (12345) (12) & $\mathrm{SP}(1)$ & 3.797 & AM \\
\hline 3 & -37.703512 & (12345) (12) & SP (1) & 3.750 & AM \\
\hline 4 & -37.634366 & (12345) (13) & SP (2) & 3.750 & AM \\
\hline 5 & -37.357585 & (12345) (12) & SP (3) & 4.750 & AM \\
\hline 6 & -37.288682 & (12345) (14) & SP (4) & 4.740 & AM \\
\hline 7 & -37.285022 & (12345) (12) & SP (4) & 3.750 & AM \\
\hline 8 & -36.737326 & (12346) (16) & SP (5) & 3.750 & AM \\
\hline
\end{tabular}

Interatomic distance $R e=1.1380338 \AA$. Total energy is given in Hartrees. Occupancy means MO occupancy scheme-in the first parentheses, alpha molecular spin-orbital occupancy, and in the second parentheses, beta molecular spin-orbital occupancy. The ASDW stability matrix is used to characterize the extremum. The notation $\operatorname{SP}(X)$ means a saddle point with $X$ stability matrix eigenvalues less than zero.

have used the pure aufbau SCF procedure only. Using HFPN equations and the ASDW stability matrix, we obtained seven solutions, where four are ASDW (nonsinglet) solutions and three are TICS (singlet) solutions. Only one solution is a minimum and only one is a minimum*; all other solutions are saddle points, this fact again contradicts Facelli and Contreras' theoretical results [11]. An RHF calculation using a pure SCF aufbau procedure was given the same three singlet solutions, presented in Table IX, but in this case, using the TICS stability matrix, one is identified as a minimum* and two as saddle points, instead of three ASDW saddle points. The solution classified as a minimum*, in the TICS class, was found only after a careful choice of the

\section{TABLE VIII}

Multiple UHF solutions for $C_{2}$, with $\left\langle S_{z}\right\rangle=\mathbf{0 . 0}$.

\begin{tabular}{clll}
\hline Solution & Total energy & Extremum & $\left\langle S^{2}\right\rangle$ \\
\hline 1 & -75.302267 & Minimum & 1.780 \\
2 & -75.245462 & Minimum* & 1.236 \\
3 & -75.214829 & SP (1) & 1.067 \\
4 & -75.178787 & SP (2) & 1.0 \\
5 & -75.162885 & SP (3) & 0.0 \\
6 & -75.162719 & SP (5) & 0.0 \\
7 & -75.136109 & SP (4) & 0.0 \\
\hline
\end{tabular}

Interatomic distance $R e=1.2337470 \AA$ and accuracy less than $10^{-8}$ in the density matrix. All calculations were performed with the pure SCF aufbau procedure only. Total energy is given in Hartrees. The ASDW stability matrix is used to characterize the extremum. The notation $\mathrm{SP}(\mathrm{X})$ means a saddle point with $\mathrm{X}$ stability matrix eigenvalues less than zero. starting LCAO coefficients for the pure SCF procedure. We found that the $\mathbf{Q}$ Stanton's matrices for TICS solutions 2 and 3, classified as saddle points (see Table IX), present 2 and 1 eigenvalues greater than 1, respectively. The existence of these eigenvalues greater than 1 does not implicate in a numerical intrinsic divergence in the aufbau SCF procedure. These results are in accordance with the general equation (2.10) found by Facelli and Contreras but are in disagreement with their conclusion, that is, that the convergence of a pure aufbau SCF procedure implicates, necessarily, that the found solution is a minimum in its respective class.

In Table $X$, we present multiple UHF/HFPN solutions for $\mathrm{N}_{2}$, with $\left\langle S_{z}\right\rangle=0.0$. They were calculated using the pure SCF aufbau procedure, interatomic distance $\operatorname{Re}=1.1366370 \AA$, and accuracy less than $10^{-8}$ in the density matrix. In this case, we obtained eight solutions, where five are ASDW (nonsinglet)

TABLE IX Multiple RHF solutions for $C_{2}$, with $\left\langle S_{z}\right\rangle=0.0$.

\begin{tabular}{clll}
\hline Solution & Total energy & Extremum & $\left\langle S^{2}\right\rangle$ \\
\hline 1 & -75.162885 & Minimum* & 0.0 \\
2 & -75.162719 & SP (2) & 0.0 \\
3 & -75.136109 & SP (1) & 0.0
\end{tabular}

Interatomic distance $R e=1.2337470 \AA$ and accuracy less than $10^{-8}$ in the density matrix. All calculations were performed with the pure SCF aufbau procedure only. Total energy is given in Hartrees. The TICS stability matrix is used to characterize the extremum. The notation $\operatorname{SP}(X)$ means a saddle point with $X$ stability matrix eigenvalues less than zero. 
TABLE X

Multiple UHF solutions for $\mathrm{N}_{2}$, with $\left\langle S_{z}\right\rangle=\mathbf{0 . 0}$.

\begin{tabular}{clll}
\hline Solution & Total energy & Extremum & $\left\langle S^{2}\right\rangle$ \\
\hline 1 & -108.548190 & Minimum & 0.182 \\
2 & -108.547232 & SP (1) & 0.0 \\
3 & -108.275283 & SP (1) & 1.148 \\
4 & -108.260206 & SP (2) & 1.0 \\
5 & -108.053861 & SP (2) & 2.0 \\
6 & -107.937910 & SP (3) & 1.108 \\
7 & -107.876369 & SP (7) & 0.0 \\
8 & -107.866282 & SP (6) & 0.0 \\
\hline
\end{tabular}

Interatomic distance $R e=1.1366370 \AA$ and accuracy less than $10^{-8}$ in the density matrix. All calculations were performed with the pure SCF aufbau procedure only. Total energy is given in Hartrees. The ASDW stability matrix is used to characterize the extremum. The notation SP $(X)$ means a saddle point with $X$ stability matrix eigenvalues less than zero.

solutions and three are TICS (singlet) solutions. Only one solution is a minimum and all other solutions are saddle points. As in the case of $C_{2}$ system, this result is in disagreement with Facelli and Contreras affirmation. An RHF calculation gave the same three singlet solutions (Table XI), and using the TICS stability matrix, one is identified as a minimum and the other two as saddle points, instead of three saddle points obtained using the ASDW stability matrix. A calculation of the $\mathbf{Q}$ Stanton's matrix eigenvalues for RHF/TICS solutions presented in Table XI shows that solution 1 has $\left|\lambda_{\max }\right|<1$ and solutions 2 and 3 have two eigenvalues greater than the one associated to their respective $\mathbf{Q}$ matrix; this fact, however, does not implicate an intrinsic numerical divergence. The existence of $\mathbf{Q}$ eigenvalues with $\left|\lambda_{\max }\right|>1$ in pure converged SCF procedures does not signify that our results are in disagreement

TABLE XI

Multiple RHF solutions for $\mathbf{N}_{\mathbf{2}}$, with $\left\langle S_{\mathbf{z}}\right\rangle=\mathbf{0 . 0}$.

\begin{tabular}{clll}
\hline Solution & Total energy & Extremum & $\left\langle S^{2}\right\rangle$ \\
\hline 1 & -108.547232 & Minimum & 0.0 \\
2 & -107.876369 & SP $(2)$ & 0.0 \\
3 & -107.866282 & SP (2) & 0.0
\end{tabular}

Interatomic distance $R e=1.1366370 \AA$ and accuracy less than $10^{-8}$ in the density matrix. All calculations were performed with the pure SCF aufbau procedure only. Total energy is given in Hartrees. The TICS stability matrix is used to characterize the extremum. The notation SP $(X)$ means a saddle point with $\mathrm{X}$ stability matrix eigenvalues less than zero. with Stanton's criterion for intrinsic numerical convergence. The explanation why we do not observe, in these cases, an intrinsic divergence is directly connected with the symmetry considerations made by Stanton and commented on by us in Section 2.2.

\section{Conclusions}

In the study of convergence criteria for the classical SCF procedure, it is known in the literature that (i) the SCF procedure is intrinsically numerical convergent if $\left|\lambda_{\max }\right|<1$, where $\lambda_{\max }$ is the largest eigenvalue in the absolute value of the matrix $\mathbf{Q}$ introduced by Stanton [9]; (ii) if the classical SCF algorithm converges, then one can be sure that the corresponding closed-shell energy is a minimum in the allowed space of singlet functions [10]; and (iii) the two statements above can be extended for a general one-determinantal wave function which satisfies the aufbau principle [11]. To verify these theoretical criteria, we make some calculations on $\mathrm{H}_{2}, \mathrm{LiH}, \mathrm{CH}, \mathrm{C}_{2}$, and $\mathrm{N}_{2}$ systems. An analysis of our results (see Tables I-XI) shows that the pure SCF iterative procedure with the aufbau scheme can converge to solutions classified as saddle points. As a consequence of our results, we can conclude that the Facelli and Contreras affirmation, that is, that in a pure SCF converged aufbau calculation, the solution is necessarily a minimum, is not valid in general. It is important to make some comments about in what context we disagree with the conclusion cited above. First, we agree that in a pure SCF converged aufbau calculation the solution obtained will be a minimum in a certain subspace of Fukutome's classes of one-determinantal functions (where only restrictions upon spin are considered). The subspace in question is determined by the additional constraints used, implicitly or not, in the variational process used to determine the converged solution. To achieve this conclusion that the final solution is a minimum, it is necessary that, in Eq. (2.10), the stability matrix E considers all the constraints included in the variational process. In a pure SCF aufbau procedure, without an explicit spacial symmetry restriction, the implicit constraints included in the variational process are directly connected with the symmetries contained in the MOs taken as an initial guess. In this way, we can consider that the conclusion of Contreras and Facelli is valid, in general, if one assumes that the initial guesses have a complete symmetry breaking in their MOs. In this sense, our numerical study reinforces Löwdin's 
standpoint [16] that it is essential to understand in greater detail the connection between the final SCF solution and the starting point of the SCF procedure. In the context of multiple solutions, it is natural that we characterize these solutions in the same space or subspace. Fukutome's classes TICS and ASDW, and their respective stability matrices, are natural ones for RHF and UHF calculus, respectively. Our results show that although we can consider all HF solutions, obtained by a pure $\mathrm{SCF}$ aufbau procedure, as minimum points in their respective subspaces, when evaluated in the same space (associated to one of Fukutome's eight classes and where only constraints upon spin are imposed), the change to saddle points is a behavior that we cannot consider as an exception. This result is not obvious principally if we consider that very few minima were found for all systems, considering both TICS and ASDW classes of solutions. In light of our results, we can cite two advantages in evaluating all the multiple HF solutions in the same functional space: (i) detection of a poor initial guess, or the presence of another solution with lower energy, by the inspection of a saddle-point character in the converged solution. This can be useful when the system has not a special symmetry; (ii) in the calculus of dissociation curves, the detection of saddle points, especially by an analysis of the number of eigenvalues less than zero, can be useful to distinguish solutions pertaining to different curves in regions where two or more curves are very close or crossing.

Relative to solutions classified as a local minimum point, we obtained the following results: For $\mathrm{H}_{2}$, we obtained, for $\left\langle S_{z}\right\rangle=0.0$, only one minimum in each class (TICS and ASDW) of solutions; for the $\mathrm{LiH}$ and $\mathrm{CH}$ systems, we obtained, to each set of solution characterized by a specific value for $\left\langle S_{z}\right\rangle$, only one minimum relative to the ASDW class; for the $C_{2}$ system, we obtained one minimum relative to the TICS class and two minima relative to the ASDW class of solutions; and for $\mathrm{N}_{2}$, we obtained only one minimum in each class of solutions. In all cases, the minimum points correspond to an aufbau orbital occupancy scheme. We did not find any solution classified as a minimum point with an energy value greater than a solution classified as a saddle point, but we found non-aufbau solutions, for the $\mathrm{CH}$ system (see Tables VI and VII), with an energy lower than aufbau solutions pertaining to the same ASDW class and with a same value for $\left\langle S_{z}\right\rangle$. All solutions that we have found with non-aufbau orbital occupancy schemes are saddle points and have been determined mainly with the AM.
Our results, principally those related to the $\mathrm{CH}$ and $\mathrm{C}_{2}$ systems, show that the pursuit for a solution classified as a minimum extremum point of the HF functional in its respective class of functions can be a very difficult task. This situation corresponds to finding a solution associated to a numerical intrinsic divergence and with some of the $\mathbf{Q}$ Stanton's matrix eigenvalues less than -1 (and $\left|\lambda_{i}\right|<1$ for the others). In some cases, the only way to achieve such a solution using an iterative procedure like the SCF method implies the utilization of algorithms to enforce the convergence in conjunction or/and a careful choice of the starting MOs. A pure aufbau SCF procedure does not assure that a convergent solution will be a minimum extremum point with respect to the TICS or ASDW classes and a stability analysis is necessary to characterize the solution. It is important to point out that there is no criterion described in the literature which allows us to distinguish a local minimum from a global one without an extensive investigation of the hypersurface associated to the respective HF functional. The problem of finding the absolute minimum remains open.

Finally, due to the nonlinear character of the integrodifferential system of equations relative to the HF functional, it is expected that different basis sets, for a same system at the same geometry, will be associated with different hypersurfaces which can differ in the number and type of extremum points. However, in any basis set, it is possible to obtain similar results as presented here.

\section{ACKNOWLEDGMENTS}

The authors are grateful to Professor M. A. C. Nascimento for his interest in this work and very useful suggestions. One of the authors (L. E. D.) thanks the CNPq and JDMV thanks CAPES for financial support.

\section{References}

1. Thouless, D. J. The Quantum Mechanics of Many-Body Systems; Academic: New York, 1961.

2. Adams, W. H. Phys Rev 1962, 127, 1650.

3. Mayer, I.; Löwdin, P. O. Chem Phys Lett 1993, 202, 1.

4. Cizek, P.; Paldus, J. J Chem Phys 1967, 47, 3976.

5. Löwdin, P. O.; Calais, J. L.; Calazans, J. M. Int J Quantum Chem 1981, 20, 1201.

6. Fukutome, H. Int J Quantum Chem 1981, 20, 955.

7. Silva, G. M.; Malbouisson, L. A. C.; Naves de Brilō, A.; Vianna, J. D. M. J Math Chem 1993, 13, 317. 


\section{DARDENNE ET AL.}

8. Silva, G. M.; Malbouisson, L. A. C.; Vianna, J. D. M. J Math Chem 1995, 17, 347.

9. Stanton, R. E. J Chem Phys 1981, 75, 5416.

10. Facelli, J. C.; Contreras, R. H. J Chem Phys 1982, 77, 1076.

11. Facelli, J. C.; Contreras, R. H. J Chem Phys 1983, 79, 3421.

12. Malbouisson, L. A. C.; Vianna, J. D. M. J Chim Phys 1990, 87, 2017.
13. Teixeira Filho, R. M.; Malbouisson, L. A. C.; Vianna, J. D. M. J Chim Phys 1993, 90, 1999.

14. Schmidt, M. W.; Baldrige, K. K.; Boatz, J. A.; Elbert, S. T.; Gordon, M. S.; Jensen, J. H.; Koseki, S.; Matsunaga, N.; Nguyen, K. A.; Su, S. J.; Windus, T. L.; Dupuis, M.; Montgomery, J. A. J Comp Chem 1993, 14, 1347.

15. Brown, K. M.; Siam, J. Num Anal 1969, 6, 560.

16. Löwdin, P. O.; Mayer, I. Adv Quantum Chem 1992, 24, 79. 